\title{
Role of tumour necrosis factor stimulated gene 6 (TSG-6) in the coupling of inter- $\alpha$-trypsin inhibitor to hyaluronan in human follicular fluid
}

\author{
T. E. Jessen ${ }^{1}$ and L. Ødum ${ }^{2 *}$ \\ ${ }^{1}$ Department of Clinical Biochemistry, Holbæk, Sygehus Vestsjælland, DK-4300 Holbæk, Denmark; and \\ ${ }^{2}$ Department of Clinical Biochemistry, Roskilde University Hospital, DK-4000 Roskilde, Denmark
}

\begin{abstract}
Binding of the plasma proteinase inhibitor inter- $\alpha$-trypsin inhibitor (ITI) to hyaluronan is necessary for normal expansion of the cumulus-oocyte complex. Lack of ITI causes severe infertility. Binding of ITI to hyaluronan depends on calcium ions and coupling activity present in follicular fluid (Ødum et al., 2002). The complexes formed by this process contain ITI heavy chains bound to hyaluronan, and bikunin is detached from ITI during the coupling reaction. In the present study, an electrophoretic technique by which hyaluronan-bound ITI is immobilized was used to demonstrate that tumour necrosis
\end{abstract}

factor stimulated gene 6 (TSG-6) is necessary for the coupling reaction. Thus, immunoprecipitation of TSG-6 in human follicular fluid eliminates the coupling reaction and re-addition restores the activity. However, it appears that components other than hyaluronan, ITI, calcium ions and TSG-6 are involved in the coupling reaction, as in vitro incubation of these components does not generate stable complexes between ITI heavy chains and hyaluronan unless some follicular fluid is added. In conclusion, TSG-6 is necessary for the coupling of ITI to hyaluronan, but at least one additional component in follicular fluid is essential.

\section{Introduction}

The cumulus-oocyte complex (COC) undergoes a profound expansion before ovulation in response to the mid-cycle surge of gonadotrophins. This expansion is important for normal ovulation and collection of the COC by the oviduct (Salustri et al., 1989; Hess et al., 1999; Lam et al., 2000). The ovulatory surge induces synthesis of hyaluronan by the cumulus cells (Chen et al., 1993) and an increased permeability of the blood-follicle barrier leading to an influx of plasma-derived proteins including inter- $\alpha$-trypsin inhibitor (ITI) (Powers et al., 1995; Hess et al., 1998). Furthermore, LH appears to induce formation of a coupling activity that supports the binding of ITI to hyaluronan (Ødum et al., 2002). The formed complexes stabilize the extracellular matrix (Chen et al., 1992, 1994). Studies have shown that ITI is essential for normal reproduction. Thus, female mice lacking ITI are infertile, but infertility can be reversed by injection of ITI (Sato et al., 2001; Zhuo et al., 2001).

Human ITI consists of three different polypeptide chains named bikunin, heavy chain 1 and heavy chain 2 (Ødum, 1990; Salier et al., 1996; Fries and Blom, 2000). Bikunin, a Kunitz-type proteinase inhibitor, is covalently linked to both of the heavy chains via a chondroitin sulphate bridge (Jessen et al., 1988; Enghild et al., 1989).

*Correspondence

Email: rslaod@ra.dk
ITI is synthesized in the liver (Mizushima et al., 1998) and transported via the blood to the follicles. Upon LH stimulation, the blood-follicle barrier opens to allow influx of ITI into the follicle (Hess et al., 1998). Inside the follicle, ITI forms complexes with hyaluronan in a coupling reaction in which bikunin is detached from ITI. Thus, complexes in follicular fluid formed in vivo contain only ITI heavy chains linked to hyaluronan (Jessen et al., 1994; Eriksen et al., 1999). When the coupling reaction is performed in vitro, ITI, hyaluronan, calcium ions and at least one unidentified protein produced locally in the follicle are required for successful coupling to take place (Ødum et al., 2002).

Tumour necrosis factor-stimulated gene 6 (TSG-6) is a $35 \mathrm{kDa}$ glycoprotein. TSG- 6 gene expression has been shown to be upregulated in cumulus cells during expansion of the mouse COC in vivo (Fülöp et al., 1997) and in the ovaries $4-8 \mathrm{~h}$ after stimulation with hCG (Yoshioka et al., 2000). Immunohistochemical studies have revealed that TSG- 6 and ITI co-localize in the hyaluronan-rich matrix around cumulus cells of the expanded mouse COC (Carrette et al., 2001). A $125 \mathrm{kDa}$ firm complex between TSG- 6 and either of the heavy chains of ITI has been extracted from mouse COC by hyaluronidase digestion (Mukhopadhyay et al., 2001). This result strongly indicates that a complex between TSG- 6 and the heavy chains of ITI is bound to hyaluronan as a structural part of the COC. However, it is not known whether the binding between ITI and hyaluronan 
is dependent on an interaction with TSG-6. These observations made it relevant to investigate whether TSG-6 is involved in the coupling of ITI to hyaluronan. The purpose of this study was therefore to investigate whether TSG- 6 participates in the coupling reaction. The effect of anti-TSG- 6 antibodies on the coupling reaction was determined using immunoelectrophoresis (Ødum et al., 2002). Subsequently, TSG-6 protein was added to investigate whether activity could be restored.

\section{Materials and Methods}

\section{Reagents and chemicals}

Ovine testicular hyaluronidase (EC 3.2.1.35, specific activity 49130 national formulary (NF) units (as defined in United States Pharmacopeia) per mg protein) and human umbilical cord hyaluronic acid were obtained from Calbiochem (Novabiochem Corporation, Darmstadt). Rabbit anti-human ITI immunoglobulin directed against all peptides of ITI (that is, bikunin, heavy chain 1 and heavy chain 2) was obtained from Dako A/S (Copenhagen) (Jessen et al., 1994). Agarose LSL 4000 was obtained from FMC BioProducts (Rockland, ME). ITI was purified from serum using the method described by Jochum and Bittner (1983) and appeared on SDSPAGE as a single band with a molecular mass of 240 kDa. Recombinant TSG-6 and rabbit antibodies directed against TSG-6 were kindly provided by $\mathrm{H}-\mathrm{G}$. Wisniewski (DNYU School of Medicine, New York). Pooled preovulatory human follicular fluids were obtained as described by Andersen et al. (1999). The use of human follicular fluid for research purposes was approved by the Ethical Committee for the counties of Copenhagen and Frederiksberg, and patients gave consent after having received information orally and in writing. Immunoprecipitated human follicular fluids were obtained by addition of anti-TSG- 6 antibodies to a pool of human follicular fluid followed by incubation for $18 \mathrm{~h}$ at $4^{\circ} \mathrm{C}$, and centrifugation (14000 $\mathrm{g}$ for $15 \mathrm{~min}$ ). The amount of anti-TSG- 6 antibody added was adjusted to give a supernatant devoid of TSG-6. All other reagents and chemicals were of analytical grade.

\section{Demonstration of coupling activity by immunoelectrophoresis}

Experiments were performed by mixing the following components: (i) ITI source: one volume of normal human serum $\left(1 \mathrm{U} \mathrm{ITI} \mathrm{ml}^{-1}\right)$ or purified ITI $\left(1.5 \mathrm{U} \mathrm{ml}^{-1}\right)$, (ii) buffer: one volume of $45 \mathrm{mmol}$ Tris- $\mathrm{HCl} \mathrm{I}^{-1}, \mathrm{pH} 7.4$; $4.5 \mathrm{mmol} \mathrm{CaCl} \mathrm{I}^{-1} ; 150 \mathrm{mmol} \mathrm{NaCl}{ }^{-1} ; 4.5 \mathrm{mg}$ hyaluronan $\mathrm{ml}^{-1}$, with or without $18 \mu \mathrm{g}$ or $36 \mu \mathrm{g}$ TSG- 6 $\mathrm{ml}^{-1}$ and (iii) follicular fluid: one volume of human follicular fluid or immunoprecipitated human follicular fluid. In some experiments follicular fluid was replaced with saline. The mixtures were incubated at $37^{\circ} \mathrm{C}$.
Aliquots were withdrawn (at 0,3 and $24 \mathrm{~h}$ ), and $1.5 \mathrm{~mol} \mathrm{NaCl} \mathrm{I}{ }^{-1}$ (one third of the withdrawn volume) was added before storing at $-20^{\circ} \mathrm{C}$. The visualization of hyaluronan-bound (immobilized) heavy chains was performed by a two-step electrophoresis (Jessen et al., 1994; Ødum et al., 2001). Heavy chains bound to hyaluronan were retained in the application well during removal of unbound proteins by electrophoresis. The retained hyaluronan was then degraded and the released hyaluronan-bound proteins detected by electrophoresis in an antibody-containing gel. In step 1, separation electrophoresis of $10 \mu \mathrm{l}$ samples was carried out at $10 \mathrm{~V} \mathrm{~cm}^{-1}$ for $60 \mathrm{~min}$. After electrophoresis, sample wells were treated with testis hyaluronidase $(50 \mathrm{U}$ per well, $1 \mathrm{~h}, 37^{\circ} \mathrm{C}$ ) for detection of hyaluronan-bound protein. In step 2, after digestion of hyaluronan in sample wells, the gel at the anodic side of the wells containing the anodic migrating proteins of the first run was replaced by antiITI gel $\left(1.1 \mu \mathrm{cm}^{-2}\right)$. Electroimmunoassay of the ITI polypeptides released by hyaluronidase treatment was run overnight at $2.5 \mathrm{~V} \mathrm{~cm}^{-1}$ into the anti-ITI gel (Axelsen and Bock, 1983). All experiments were performed at least twice.

The coupling activity was expressed as the concentration of immobilized ITI heavy chains formed during incubation for $1 \mathrm{~h}\left(\mathrm{mU} \mathrm{ml}^{-1} \mathrm{~h}^{-1}\right)$, where $1 \mathrm{U}$ corresponds to the total amount of ITI heavy chains in $1 \mathrm{ml}$ normal human serum. Coupling activity was measured during the first $3 \mathrm{~h}$ of the incubation period, when the substrates ITI and hyaluronan were in excess.

\section{Results}

Involvement of TSG-6 in the coupling reaction

Coupling activity in follicular fluid (Fig. 1a, lanes 1-3) was expressed as the increase in heavy chainhyaluronan complexes formed during $24 \mathrm{~h}$ after mixing of human follicular fluid, human serum and hyaluronan. The ITI immunoprecipitates seen at $0 \mathrm{~h}$ were due to ITI-hyaluronan complexes preformed in vivo in human follicular fluid. The coupling activity was $9.6 \mathrm{mU} \mathrm{ml}^{-1}$ $\mathrm{h}^{-1}$ at the start of the incubation period (Fig. 1a, lanes 1,2). After immunoprecipitation of TSG-6 in follicular fluid, no coupling activity was detected (Fig. 1a, lanes 4-6). The immunoprecipitates did not increase in size, demonstrating that no ITI heavy chains were coupled to hyaluronan during the incubation period. However, if TSG- 6 was subsequently added to the immunopreciptated follicular fluid $\left(6 \mu \mathrm{g} \mathrm{ml}^{-1}\right.$ or $\left.12 \mu \mathrm{g} \mathrm{ml}^{-1}\right)$, the coupling activity was restored in a dose-response manner (Fig. 1a, lanes 7-12) to $14.3 \mathrm{mU} \mathrm{m}^{-1} \mathrm{~h}^{-1}$ (lanes 7,8 ) and $24.3 \mathrm{mU} \mathrm{ml}^{-1} \mathrm{~h}^{-1}$ (lanes 10,11 ), respectively. Consequently, TSG- 6 must be essential for the coupling of ITI to hyaluronan.

The coupling reaction was attempted with purified components to examine whether TSG-6 is identical 
(a)

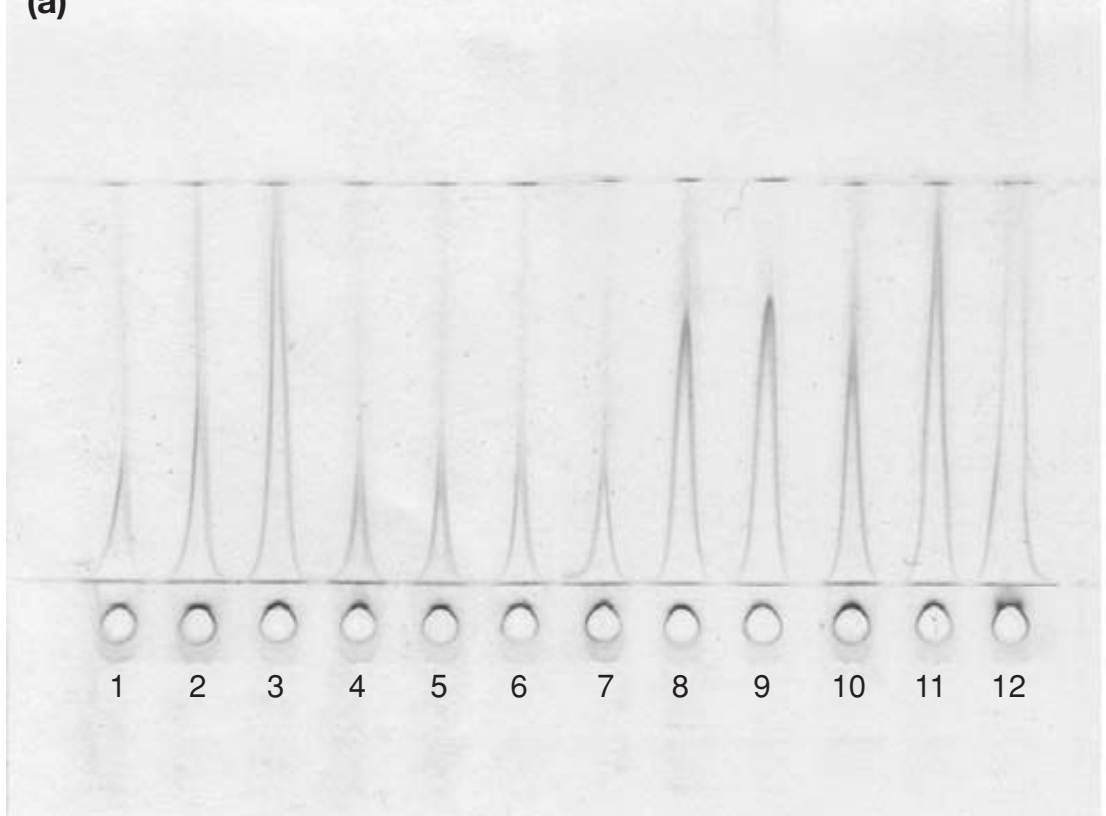

(b)

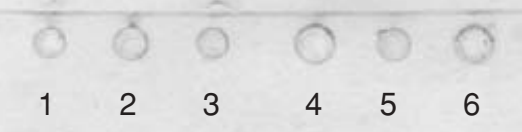

Fig. 1. Involvement of tumour necrosis factor-stimulated gene 6 (TSG-6) in the coupling reaction of inter- $\alpha$-trypsin inhibitor (ITI) to hyaluronan in human follicular fluid. Complexes between ITI and hyaluronan were demonstrated by a two-step immunoelectrophoresis in which hyaluronan and hyaluronan-bound proteins were retained in electrophoretic wells. After removal of unbound proteins, hyaluronan-bound proteins were released by enzymatic degradation of hyaluronan. (a) Coupling activity in the presence of follicular fluid. Lanes 1-3: control experiment with mixture of human follicular fluid, hyaluronan and serum incubated for 0,3 and $24 \mathrm{~h}$, respectively. Lanes 4-6: mixture of TSG-6-immunoprecipitated human follicular fluid, hyaluronan and serum incubated for 0, 3 and 24h, respectively. Lanes 7-9: mixture of TSG-6-immunoprecipitated human follicular fluid, hyaluronan and serum, with addition of $6 \mu \mathrm{g}$ TSG- $6 \mathrm{ml}^{-1}$, incubated for 0,3 and $24 \mathrm{~h}$, respectively. Lanes 10-12: mixture of TSG-6-immunoprecipitated human follicular fluid, hyaluronan and serum, with addition of $12 \mu \mathrm{g} \mathrm{TSG}-6 \mathrm{ml}^{-1}$, incubated for 0,3 and $24 \mathrm{~h}$, respectively. (b) Coupling activity with purified components without follicular fluid. Lanes 1-3: mixture of hyaluronan and ITI incubated for 0,3 and $24 \mathrm{~h}$, respectively. Lanes 4-6: mixture of hyaluronan and ITI, with addition of $12 \mu \mathrm{g} \mathrm{TSG-} 6 \mathrm{ml}^{-1}$, incubated for 0,3 and $24 \mathrm{~h}$, respectively.

to the recently proposed coupling factor in follicular fluid (Ødum et al., 2002). Hyaluronan was incubated with purified human ITI under physiological conditions with and without TSG-6. Only minute amounts of ITI bound to hyaluronan under these conditions (Fig. 1b). Thus, even though TSG-6 is necessary for the coupling of ITI to hyaluronan, it can account for only a part of the coupling activity of follicular fluid.

\section{Discussion}

Binding of ITI to hyaluronan is of great importance for normal expansion of the cumulus-oocyte complex. However, the processes leading to this binding are mainly unknown. Complexes between ITI and hyaluronan generated in vivo contain only the heavy chains of ITI (Jessen et al., 1994), so a detachment of bikunin seems to take place in connection with the binding of ITI to hyaluronan. Experiments in vitro have shown that complexes between ITI and hyaluronan contain bikunin shortly after their formation, but that bikunin is absent in the complexes after $24 \mathrm{~h}$ (Ødum et al., 2002). Thus, the initial binding of ITI to hyaluronan seems to be possible when bikunin is still part of the ITI molecule.

Human and bovine follicular fluids contain unidentified protein factors that are necessary for the binding of ITI heavy chains to hyaluronan in vitro (Ødum et al., 2002). A factor secreted by granulosa cells possesses the same properties (Chen et al., 1996). Thus, a mixture of purified ITI and hyaluronan alone does not generate stable complexes between ITI heavy chains and hyaluronan. Follicular fluid containing the unidentified coupling activity has to be added (Ødum et al., 2002).

TSG-6, which can bind both ITI and hyaluronan (Wisniewski et al., 1994), is synthesized in the follicle by the cumulus cells, and its gene expression is upregulated during expansion of mouse COC in vivo (Fülöp et al., 1997). Complexes between TSG-6, ITI heavy chains and hyaluronan have been detected in the hyaluronan-rich matrix around the cumulus cells of the expanded mouse COC (Carrette et al., 2001). Therefore, TSG-6 was considered to be a possible coupling factor involved in the physiological binding of ITI to hyaluronan. 
The present study has shown that the coupling activity of human follicular fluid disappears when endogenous TSG-6 is immunoprecipitated. Furthermore, coupling activity can be restored in a dose-response manner by addition of exogenous TSG- 6 to the immunoprecipitated follicular fluid. Consequently, TSG- 6 must be essential for the coupling of ITI to hyaluronan. This result is in accordance with the observation that the coupling activity in follicular fluid increases after the mid-cycle surge of gonadotrophins (Ødum et al., 2002). This occurs simultaneously with the upregulation of TSG-6 gene expression (Fülöp et al., 1997, Yoshioka et al., 2000). The concentration of TSG-6 in follicular fluid has, to the authors' knowledge, not been described, but concentrations of up to $20 \mu \mathrm{g} \mathrm{ml}^{-1}$ have been detected in synovial fluid from patients with arthritis (Wisniewski et al., 1993). In the present study, similar concentrations were effective in vitro, $12 \mu \mathrm{g} \mathrm{ml}^{-1}$ giving a higher response than $6 \mu \mathrm{g} \mathrm{ml}^{-1}$.

Although TSG- 6 is necessary for the normal coupling of ITI to hyaluronan, it is not sufficient. Thus, a mixture of purified components (including calcium ions) does not generate ITI-hyaluronan complexes.

In conclusion, TSG-6 is necessary for the coupling of ITI to hyaluronan, but at least one additional component in follicular fluid essential for the coupling reaction remains to be identified.

The authors wish to thank C. Y. Andersen (Laboratory of Reproductive Biology, Rigshospitalet, University of Copenhagen, Blegdamsvej 9, DK-2100 Copenhagen, Denmark) for donation of follicular fluids and H-G. Wisniewski for donation of recombinant TSG-6 and TSG-6 antibody.

\section{References}

Andersen CY, Morineau G, Fukuda M, Westergaard LG, Ingerslev HJ, Fiet J and Byskov AG (1999) Assessment of the follicular cortisol: cortisone ratio Human Reproduction 14 1563-1568

Axelsen NH and Boch E (1983) Electroimmunoassay (rocket immunoelectrophoresis) Scandinavian Journal of Immunology 17 103-106

Carrette O, Nemade RV, Day AJ, Brickner A and Larsen WJ (2001) TSG-6 is concentrated in the extracellular matrix of mouse cumulusoocyte complexes through hyaluronan and inter-alpha-inhibitor binding Biology of Reproduction 65 301-308

Chen L, Mao SJ and Larsen WJ (1992) Identification of a factor in fetal bovine serum that stabilizes the cumulus extracellular matrix. A role for a member of the inter-alpha-trypsin inhibitor family Journal of Biological Chemistry 26712 380-12 386

Chen L, Russel PT and Larsen WJ (1993) Functional significance of cumulus expansion in the mouse: role for the preovulatory synthesis of hyaluronic acid within the cumulus mass Molecular Reproduction and Development 34 87-93

Chen L, Mao SJ, MacLean LR, Powers RW and Larsen WJ (1994) Proteins of the inter-alpha-trypsin inhibitor family stabilize the cumulus extracellular matrix through their direct binding with hyaluronic acid Journal of Biological Chemistry 26928 282-28 287

Chen L, Zhang H, Powers RW, Russell PT and Larsen WJ (1996) Covalent linkage between proteins of the inter-alpha-inhibitor family and hyaluronic acid is mediated by a factor produced by granulosa cells Journal of Biological Chemistry 27119 409-19414
Enghild JJ, Thogersen IB, Pizzo SV and Salvesen G (1989) Analysis of inter-alpha-trypsin inhibitor and a novel trypsin inhibitor, pre-alphatrypsin inhibitor, from human plasma. Polypeptide chain stoichiometry and assembly by glycan Journal of Biological Chemistry $\mathbf{2 6 4}$ 15 975-15981

Eriksen GV, Carlstedt I, Mörgelin M, Uldbjerg N and Malmstrøm A (1999) Isolation and characterization of proteoglycans from human follicular fluid Biochemical Journal 340 613-620

Fries E and Blom AM (2000) Bikunin - not just a plasma proteinase inhibitor International Journal of Biochemistry and Cell Biology 32 $125-137$

Fülöp C, Kamath RV, Li Y, Otto JM, Salustri A, Olsen BR, Glant TT and Hascall VC (1997) Coding sequence, exon-intron structure and chromosomal localization of murine TNF-stimulated gene 6 that is specifically expressed by expanding cumulus cell-oocyte complexes Gene 202 95-102

Hess KA, Chen L and Larsen WJ (1998) The ovarian blood-follicle barrier is both charge- and size-selective in mice Biology of Reproduction $\mathbf{5 8}$ 705-711

Hess KA, Chen L and Larsen WJ (1999) Inter-alpha-inhibitor binding to hyaluronan in the cumulus extracellular matrix is required for optimal ovulation and development of mouse oocytes Biology of Reproduction 61 436-443

Jessen TE, Faarvang KL and Ploug M (1988) Carbohydrate as covalent crosslink in human inter-alpha-trypsin inhibitor: a novel plasma protein structure FEBS Letters 230 195-200

Jessen TE, Ødum L and Johnsen AH (1994) In vivo binding of human interalpha-trypsin inhibitor free heavy chains to hyaluronic acid Biological Chemistry Hoppe Seyler 375 521-526

Jochum M and Bittner A (1983) Inter-alpha-trypsin inhibitor of human serum: an inhibitor of polymorphonuclear granulocyte elastase Hoppe Seyler's Zeitschrift für Physiologische Chemie 364 1709-1715

Lam X, Gieseke C, Knoll M and Talbot P (2000) Assay and importance of adhesive interaction between hamster (Mesocricetus auratus) oocytecumulus complexes and the oviductal epithelium Biology of Reproduction 62 579-588

Mizushima S, Nii A, Kato K and Uemura A (1998) Gene expression of the two heavy chains and one light chain forming the inter-alpha-trypsininhibitor in human tissues Biological and Pharmaceutical Bulletin 21 167-169

Mukhopadhyay D, Hascall VC, Day AJ, Salustri A and Fulop C (2001) Two distinct populations of tumor necrosis factor-stimulated gene6 protein in the extracellular matrix of expanded mouse cumulus cell-oocyte complexes Archives of Biochemistry and Biophysics 394 173-181

Ødum L (1990) Inter-alpha-trypsin inhibitor: a plasma proteinase inhibitor with a unique chemical structure International Journal of Biochemistry 22 925-930

Ødum L, Jessen TE and Andersen CY (2001) Glycosaminoglycan-bound and free inter-alpha-trypsin inhibitor components of follicular fluid Zygote 9 283-288

Ødum L, Andersen CY and Jessen TE (2002) Characterization of the coupling activity for the binding of inter- $\alpha$-trypsin inhibitor to hyaluronan in human and bovine follicular fluid Reproduction $\mathbf{1 2 4}$ 249-257

Powers RW, Chen L, Russell PT and Larsen WJ (1995) Gonadotropinstimulated regulation of blood-follicle barrier is mediated by nitric oxide American Journal of Physiology 269 E290-E298

Salier JP, Rouet P, Raguenez G and Daveau M (1996) The inter-alphainhibitor family: from structure to regulation Biochemical Journal 315 $1-9$

Salustri A, Yanagishita M and Hascall VC (1989) Synthesis and accumulation of hyaluronic acid and proteoglycans in the mouse cumulus cell-oocyte complex during follicle-stimulating hormone-induced mucification Journal of Biological Chemistry 26413 840-13 847

Sato H, Kajikawa S, Kuroda S et al. (2001) Impaired fertility in female mice lacking urinary trypsin inhibitor Biochemical and Biophysical Research Communications 281 1154-1160 
Wisniewski HG, Maier R, Lotz M, Lee S, Klampfer L, Lee TH and Vilcek J (1993) TSG-6: a TNF-, IL-1-, and LPS-inducible secreted glycoprotein associated with arthritis Journal of Immunology 151 6593-6601

Wisniewski HG, Burgess WH, Oppenheim JD and Vilcek J (1994) TSG-6, an arthritis-associated hyaluronan binding protein, forms a stable complex with the serum protein inter-alpha-inhibitor Biochemistry 33 7423-7429

Yoshioka S, Ochsner S, Russell DL, Ujioka T, Fujii S, Richards JS and Espey LL (2000) Expression of tumor necrosis factor-stimulated gene- 6 in the rat ovary in response to an ovulatory dose of gonadotropin Endocrinology 141 4114-4119
Zhuo L, Yoneda $M$, Zhao $M$, Yingsung $W$, Yoshida $N$, Kitagawa $Y$, Kawamura K, Suzuki T and Kimata K (2001) Defect in SHAP-hyaluronan complex causes severe female infertility. A study by inactivation of the bikunin gene in mice Journal of Biological Chemistry 276 7693-7696

Received 17 July 2002.

First decision 27 August 2002.

Revised manuscript received 4 September 2002.

Accepted 16 September 2002. 